\title{
Performance Analysis of Transparent Relays in 802.16j MMR Networks
}

\author{
Vasken Genc, Seán Murphy, John Murphy \\ UCD School of Computer Science and Informatics \\ University College Dublin, Belfield, Dublin 4, Ireland \\ vasken.genc@ucdconnect.ie
}

\begin{abstract}
A simulation study of the performance of transparent mode relay-based 802.16j systems is described. The study focuses on the gain in throughput that is possible in omnidirectional relay systems and increases in signalling requirements that arise due to the multihop network architecture. The study finds that the introduction of transparent mode relays cannot deliver improved throughput for a substantial part of the coverage area of a Base Station (BS) - only approximately half of the total area covered by the BS can benefit from throughput enhancement. Further, the study shows that 802.16 j based systems require significant signalling overhead, with almost twice that of 802.16e in the cases studied. Finally, the study demonstrates that in a topology with users uniformly distributed within the BS cell coverage, the maximum throughput increase on the downlink that can be achieved is relatively low. Much further work is necessary to realise useful gains from these systems.
\end{abstract}

Keywords-802.16j MMR, transparent multihop relay, performance, omnidirectional antenna.

\section{INTRODUCTION}

IEEE 802.16-based technologies are currently the subject of much interest within the community. There is much activity relating to this technology in the commercial word, with operators rolling out systems which are compliant with current versions of the standards [1] [2] - early service offerings are appearing. However, the number of subscribers is still small and there will be much growth in coming years as the technology evolves.

While the commercial world is addressing one set of problems, the research community is focusing on longer term issues. There is much activity within the research community to resolve issues with the current technologies, making them more efficient and/or easier to work with. One 802.16-related issue which is the subject of much attention right now is how to realise 802.16-based relay network solutions which are compatible with legacy equipment. That is the context for this work.

Relay network architectures can provide significant benefits over classical cellular architectures (see Figure 1). A key motivation for such systems is that they can be leveraged to realise a much lower cost network roll-out than a BS-only solution, particularly in the early stages of network deployments: this is so due to the low price point of the relays. While this is a very significant, compelling use case, there are others which are also interesting. They can be used to provide a solution for coverage holes or shadows of buildings where coverage is poor; they can be used to increase the capacity of a cell and they can be used to increase the range of cell.

The 802.16 standardisation activity is expending significant energy on issues relating to relay networks. In particular, the 802.16j [3] activity is focused on developing a relay based solution which offers mobility support and is backwards compatible with earlier 802.16 Subscriber Stations (SSs)/Mobile Stations (MSs). The standardisation activity is in the mid-stages of its development with much work remaining. At this stage, there are many open issues regarding good $802.16 j$ relay network solutions. That is the context for this work.

While there have been a few initial studies on IEEE 802.16j MMR networks, not much is really known about the performance of such systems. In particular, it is very unclear what the capacity of such systems is for different configurations. This makes it very difficult to have a clear understanding of the potential of this technology in general, and how to construct a business case based on this technology in particular.

The objective of this work, then, is to answer some initial questions regarding the performance gains that can be achieved through use of relay architectures. The study has two key aspects: firstly, there is an analysis of the impact of the location of the RS on the capacity increases that are possible and secondly, there is an analysis of the signalling that arises within such systems and a comparison with signalling in single-hop architectures.

The paper is structured as follows. Section 2 reviews related work. The current draft of IEEE 802.16j [4] is described in section 3. The simulation tools used in this work are discussed in section 4, with a reasonably detailed description of the $802.16 \mathrm{j}$ capability we have developed. Next, in section 5 , a number of simulation scenarios are described and simulation results are discussed. Finally, the paper is concluded in section 6.

\section{RELATED WORK}

At present, little research work has been carried out specifically on $802.16 \mathrm{j}$ MMR system. In this section an overview of the different publications on $802.16 \mathrm{j}$ is given. 


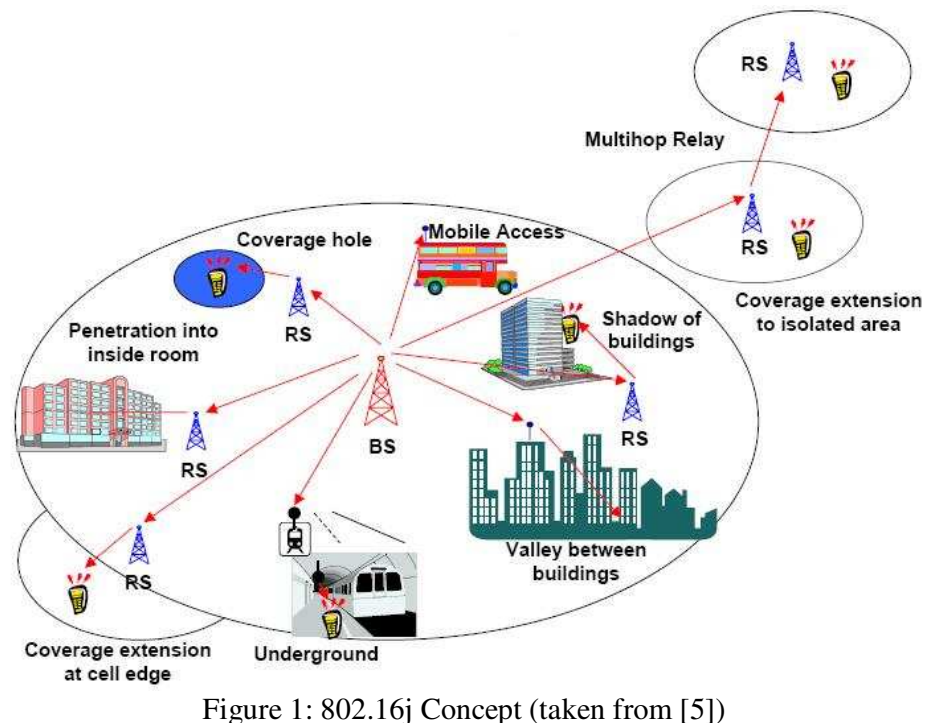

Figure 1: 802.16j Concept (taken from [5])

More specifically, related work can be divided into two sections. Firstly, some research work on the resource allocation in $802.16 \mathrm{j}$ systems is discussed. This is followed by a discussion of some work related to the design of WiMAX relay-based system intending to optimise the achievable throughput enhancement.

Proposals on resource allocation issues with $802.16 \mathrm{j}$ are presented in [6] and [7]. In both papers different schemes are proposed to optimise the MAC efficiency in such relay system. Authors in [6] propose a new downlink bandwidth allocation algorithm. This algorithm is designed to dynamically allocate resources on the downlink for RSs transmission based on the per frame bandwidth requirement. In this work, however, Orthogonal Frequency Division Multiple Access (OFDMA) is not considered and the issues relative to scheduling are not discussed at all. In [7], authors propose an aggregation scheme for traffic on the relay links (i.e., BS/RS or RS/RS links) to reduce the system overhead and thus improve the efficiency of utilisation of the MAC frame. This work highlights the necessity of connections and/or packets aggregation to avoid significant overhead in $802.16 \mathrm{j}$ system. However, it is worth noting that in the latest specifications of $802.16 \mathrm{j}$ different schemes from those defined in [7] are advocated to aggregate the traffic on the relay links.

Some initial studies on the design of $802.16 \mathrm{j}$ system have been carried out in [8] and [9]. In [8], authors present a numerical study case to find the optimal location of a relay to maximise the overall system throughput within the MR-BS cell coverage. Their work is limited to the deployment of a unique relay station and do not address the issues relative to the planning of multiple relays. Further, the system under study is based on Time Division Multiple Access/Code Division Multiple Access (TDMA/CDMA) rather than OFDMA as specified for the $802.16 \mathrm{j}$ systems. In [9], authors investigate the resource scheduling in an urban environment with high shadowing. A system with 4 directional antennas the base station and relay station is assumed. The simulations show the advantages of directional antennas over omnidirectional antennas in an urban environment. More specifically, the proposed resources scheduling methods with directional antenna show that the system throughput can be increased by 6 or 12 times compared to an omnidirectional system. This work highlights the importance of spatial reuse to maximise the throughput enhancement. The use of directional antennas leads to less interference among relays and thus more aggressive spatial reuse.

No previous work has specifically analysed the performance of $802.16 \mathrm{j}$ systems using the latest specifications provided in [4] by the 802.16 relay task group for the future 802.16 standard. More specifically, the focus of this contribution is on the analysis of the throughput performance of $802.16 \mathrm{j}$ transparent relay system described in the following section.

\section{IEEE 802.16J MMR}

Some of the key ideas of the standard as specified in [4] are becoming clear and as it is unlikely that these specifications will change dramatically, it is possible to anticipate what the new standard will look like.

In this section, the essential aspects of the 802.16j draft specifications are described. The discussion will focus on two specific aspects of the draft - the different relay modes that are defined and the frame structure that is proposed. Each of these is discussed in the following sections.

\section{A. Relay Modes}

Two different kinds of relay modes are defined: transparent mode and non-transparent mode. The key difference between these two modes of operation lies in the transmission of framing information. While devices operating in transparent mode rely on the MR-BS (Multihop Relay BS) to transmit such information, in the non-transparent mode these information are transmitted by the RSs. This leads to different benefits depending on the relays operating mode. More specifically, the transparent relays do not provide any coverage extension and aim to enhance the system throughput within the MR-BS cell coverage. The non-transparent relays, on the other hand, 
provide coverage extension while the transmission of the framing information results in high interferences among the relays and thus, limited potential throughput enhancement. At present, $802.16 \mathrm{j}$ system only operates in either transparent mode or non-transparent mode; no specifications are provided in [4] to support a system including transparent relays and nontransparent relays. Thus, the deployment of relays is aiming to either enhance the overall system throughput within the MRBS coverage or increase the MR-BS cell coverage. Table 1 below gives a comparison between the transparent and nontransparent mode.

\section{B. Frame Structure}

The initial frame structure defined in 802.16 Point to MultiPoint (PMP) system was designed for single hop wireless networks and does not support relay capabilities. Hence, a new frame structure has to be defined to support multi-hop relay architecture. The 802.16j task group considers two different kinds of frame structure for $802.16 \mathrm{j}$ system: single and multi (super) frame structure.

As with earlier 802.16 systems, the single frame structure is divided into an uplink (UL) and downlink (DL) subframe. The DL and UL subframes are respectively further portioned into different transmission zones: one access zone for MR-BS transmissions and one or multiple relay zones for relay transmissions. In such approach, the transceiver mode of the RS switches a couple of times within the frame to transmit and receive data in the DL and in the UL. This results in different periods of time within the frame during which the RS will not be able to receive or send data. This can leads to a reduction of the system achievable throughput with many transceiver switches within the frame. The multi-frame (i.e., the super frame) frame structure is composed of single frames which can be partitioned into different transmission zones. The advantage of the multi-frame approach over the single frame approach is that the frame partitioning is limited since a RS will be assigned to transmit or receive in different single frames. This will allow less transceiver mode switching of the RS and thus a better throughput performance in three or more hops networks requiring further partitioning of the single frame. However, this can give rise to some increases in delay.

While further work is required on the specification of the multi-frame structure, the baseline document clearly defines the single frame structure. In two hops system, the use of the single frame approach is specified as it provides good delay and throughput performance. In multihop networks including more than 2 hops, it is not clear which approaches (i.e., single or multi-frame) will perform better. Neither of the approach provides good performance in all scenarios. Figure 2 below depicts the frame structure in a 2 hops relay network using only transparent relays as defined in the $802.16 \mathrm{j}$ draft.

\section{Simulation MODEL}

In order to perform the study of the transparent mode relays, it was necessary to develop a tool which can simulate 802.16j capabilities. This simulation tool was developed within the QualNet simulator. Here, the simulation model is described.
Before giving details on how the simulation model is realised, there is a brief discussion of the system architecture assumed in this work. This is followed by a brief description of the 802.16 capabilities which are part of the Advanced Wireless module in the QualNet simulator. Finally, the 802.16j capabilities added to the simulator are described.

\section{A. System Architecture}

For the initial variant of the simulation model, some of the less complex variants of the different options possible within the context of $802.16 \mathrm{j}$ were selected. More specifically, simple RSs operating in transparent mode were assumed with a MRBS which contains all the system intelligence. The proposed architecture is limited to a tree topology with fixed SS (Subscriber Station) located at a maximum of two hops away from the MR-BS and with no RS-RS communication. These characteristics of the topology are derived from the use of relays operating in the transparent mode and the associated frame structure (see Figure 2). Finally, each RS in the system has its own MAC routing table distributed by the MR-BS. An example of the system architecture is depicted in Figure 3.

\section{B. WiMAX Model in QualNet}

The WiMAX simulation model implemented in QualNet [10] is based on the IEEE 802.16-2004 and 802.16e-2005 standards. It models a half duplex TDD (Time Division Duplexing) system operating in a Point to Multi-Point (PMP) mode and the OFDMA PHY.

The MAC CS (Convergence Sublayer) is implemented and supports the classification, and mapping of the higher layer PDUs (Protocol Data Units) into appropriate MAC connections. These connections are defined by a unidirectional flow of packets with specific QoS parameters and a connection identifier (CID) and represent one of the key characteristic of 802.16 technologies.

In the MAC CPS (Common Part Sublayer), the network entry and initialisation are developed. The DL/UL subframes construction and the control over how much resources are allocated in each direction are modelled. The QoS provisioning to each connection is supported and the following scheduling services defined: Unsolicited Grant Service (UGS),

Table 1

Transparent and Non-Transparent Mode Comparison

\begin{tabular}{cll}
\hline \hline & \multicolumn{1}{c}{ Transparent RS } & \multicolumn{1}{c}{ Non-Transparent RS } \\
\hline Coverage extension & No & Yes \\
Number of hops & 2 & $\geq 2$ \\
$\begin{array}{c}\text { Inter RS cell } \\
\text { interference }\end{array}$ & NA & High \\
HO between RSs & None & Yes \\
Performance & In BS coverage: & In BS coverage: same as \\
& High & $16 \mathrm{e}$ \\
& Outer BS coverage: - & Outer BS coverage: medium \\
RS Cost & Low & High \\
Scheduling & Centralised & Centralised/distributed \\
& scheduling only & scheduling \\
\hline \hline
\end{tabular}




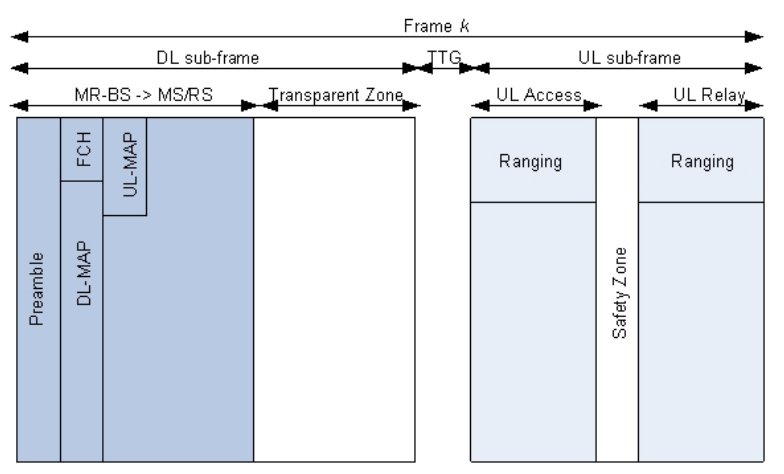

(a)

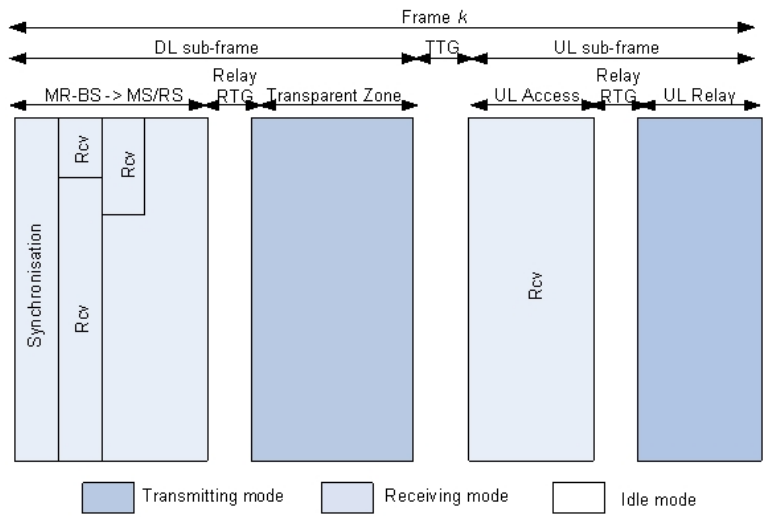

(b)

Figure 2: Transparent mode Frame Structure - (a) shows the frame structure as viewed at the MR-BS, (b) shows the frame structure as viewed at the RS

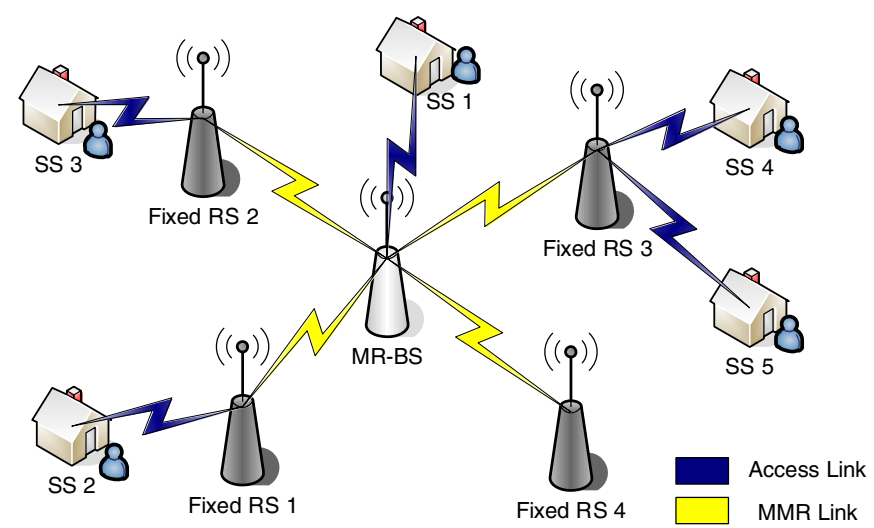

Figure 3: Example of the System Architecture

real-time Polling Service (rtPS), extended real-time Polling Service (ErtPS), non real-time Polling Service (nrtPS), and Best Effort (BE). Moreover, the bandwidth management based on a request/grant mechanism is specified. Finally, mobility support is implemented in this module although it does not support advanced capabilities such as soft handoff or sleep/idle mode.

On the PHY layer only the OFDMA PHY is modelled. An adaptive burst profile mechanism on the DL and the UL to flexibly adjust the modulation for SS is specified with the support of multiple data rate. Further, the PHY parameters such as the channel bandwidth, the FFT (Fast Fourier Transform) size, and the cyclic prefix time can be configured.

\section{Adding support for $802.16 j$ to QualNet}

To realise the $802.16 \mathrm{j}$ model some extra capabilities were added to the simulator to support relay communication. More specifically, the simulation model that was developed incorporates the following aspects of the $802.16 \mathrm{j}$ baseline document:
- modelling of the single frame structure to support transparent relay capabilities (see Figure 2);

- $\quad$ setting up of one connection between the MR-BS and $\mathrm{RS}$ for each connection between the RS and the SS;

- modelling of connection handling at the RS;

- modelling of the DL signalling on a per connection basis;

- modelling of contention based network entry procedure;

In this $802.16 \mathrm{j}$ transparent relay model, each frame is composed as follows. The DL subframe is composed of one access zone and one transparent/relay zone. The size of the relay zone is dynamically controlled to maximise the system efficiency and thus the overall system throughput. In the UL subframe, on the other hand, the relay zone size is fixed in the current implementation: this makes the current implementation less useful for studying the behaviour of the uplink. It is assumed in this model that the packets sent on the access link at destination of SSs two hops away from the MR-BS are all 
forwarded on the relay link within the same frame by the different RSs involved. Whilst the packet processing at the RSs is more likely being done over a couple of frames, this assumption does not have an impact on the system throughput performance which is the focus of this paper.

An important part of the simulation model is the implementation of a basic path decision algorithm which is left undefined in the $802.16 \mathrm{j}$ baseline document. The algorithm is used to determine how to route traffic between the SS and the MR-BS - essentially, it decides whether the traffic should be routed via a specific RS or whether there should be direct communications between SS and MR-BS. This decision is based on SS/MR-BS, SS/RS and RS/MR-BS channel conditions. The path decision algorithm determines the most efficient path based on modulation and coding schemes (MCS) used for transmission between the different nodes. Then, each RS stores its own routing table of CIDs which is updated by the MR-BS through management messages.

More specifically, this path decision is based on the following formula:

$$
\alpha_{s, r, p}=\left(\frac{W_{s}}{W_{r}+W_{p}}-1\right) * 100
$$

Where $\alpha_{s, r, p}$ denotes in percentage gain on the throughput of using the path $r+p$ instead of the path $s . W_{s}$ is a weight associated with the MR-BS/SS link, $W_{r}$ a weight associated with the MR-BS/RS link and $W_{p}$ a weight associated with the RS/SS link. Each of these weights reflects the efficiency of the links. More specifically, it represents the cost in OFDMA symbols associated to a specific MCS to transmit a bit on the medium (see Table 2).

For each SS a specific set of $\alpha_{s, r, p}$ values depending on the number of RSs are associated to it. If no value is greater than zero the SS will be directly associated to the MR-BS. Otherwise the SS will be associated to the RS corresponding to the highest $\alpha_{s, r, p}$ value. Table 2 illustrates the parameters values of the path decision algorithm.

It can be seen in Table 2 that without spatial reuse, RSs can not provide any throughput enhancement for SSs able to use the four least robust MCSs to communicate with the MR-BS. To get the maximum throughput gain shown in the table it is assumed that the RS is able to communicate with the SS and the MR-BS by using 64QAM3/4 and that no extra signalling is added.

Finally, different capabilities were omitted in the current version of the simulation model. The $802.16 \mathrm{j}$ model does not support any admission control, data fragmentation/packing, handoff mechanisms and the CDMA based ranging/bandwidth request as specified in the $802.16 \mathrm{j}$ baseline document. Further, no specific scheduling algorithm was design to map the data to specific OFDMA symbols and subchannels in the DL. The current scheduler at the MR-BS is based on the number of free PS (Physical Slot) per frame. The packets to be scheduled are chosen based on their QoS parameters and the number of free slots in the frame. It is then assumed that the scheduled data can be mapped and transmitted on the DL.

\section{RESULTS AND DisCUSSIONS}

Three specific aspects of the system performance were investigated. Firstly, some experiments were performed to determine how the RS position impacts the gain in throughput that is possible for an SS. This helps to decide where RSs should be positioned in the system. Secondly, the impact of signalling in the relay case was considered and the signalling overhead compared with single-hop 802.16 systems. Finally, there is an examination of the increase in capacity that is possible through the addition of transparent mode relays for a single MR-BS system with associated SSs. Before discussing the results, there is a short description of the simulation scenarios used.

\section{A. Simulation scenarios}

In the scenarios studied, the focus was on the system capacity in the downlink in rural environments with Single Input Single Output (SISO) omnidirectional antennas. Each scenario is comprised of a number of SSs being served by a single MR-BS where the data might be forwarded by one transparent relay. A traffic load of constant bit rate (CBR) traffic was assumed with a BE (Best Effort) scheduling service type. More specifically, the traffic load was assumed higher than the actual system capacity in the downlink to determine the maximum possible throughput increase by incorporating relays. Finally, a fair repartition of the system throughput was ensured among all the SSs and a Round-Robin scheduling algorithm was used at the base station.

In these initial experiments, the Friis free space path loss model was used. It was assumed that the signal does not suffer from any shadowing or fading effect. The transmission power was set for all stations to $30 \mathrm{dBm}$. The system was configured such that $50 \%$ of the capacity was for use in the downlink and the uplink respectively. Further, subchannel allocation in the downlink and uplink was performed with PUSC (Partial Usage of Sub-Channel) using distributed carrier permutations. Finally, from these physical parameters (see Table 3) and Hoymann's work [11] the maximum transmission range and the proportion of the entire coverage area for each MCS was derived (see Table 4).

Table 2

Path Decision Parameters

\begin{tabular}{llll}
\hline \hline Burst profile & Bit/ Symbol & $\begin{array}{c}\text { Weight } \\
(\text { Symbol/ Bit })\end{array}$ & $\begin{array}{c}\text { Maximum } \\
\text { Gain } \\
(\%)\end{array}$ \\
\hline QPSK 1/2 & 1 & 1 & 125 \\
QPSK 3/4 & $3 / 2$ & 3 & 50 \\
16QAM 1/2 & 2 & $1 / 2$ & 12.5 \\
16QAM 3/4 & 3 & $1 / 3$ & none \\
64QAM 1/2 & 3 & $1 / 3$ & none \\
64QAM 2/3 & 4 & $1 / 4$ & none \\
64QAM 3/4 & $9 / 2$ & $2 / 9$ & none \\
\hline \hline
\end{tabular}


Table 3

OFDMA PHY parameters

\begin{tabular}{lll}
\hline \hline \multicolumn{2}{c}{ OFDMA PHY parameters } & Value \\
\hline Channel Frequency $(\mathrm{GHz})$ & 3.5 \\
Transmission Power $(\mathrm{dBm})$ & 30 \\
Channel Bandwidth $(\mathrm{MHz})$ & 20 \\
FFT size & 2048 \\
Cyclic Prefix & $1 / 8$ \\
\hline \hline
\end{tabular}

Table 4

Relationship between Modulation and Coding Scheme and Coverage

\begin{tabular}{llll}
\hline \multicolumn{1}{c}{ Burst profile } & SNR & \multicolumn{1}{c}{$\begin{array}{c}\text { Transmission Range } \\
(\mathbf{k m})\end{array}$} & $\begin{array}{c}\text { Surface } \\
(\%)\end{array}$ \\
\hline QPSK 1/2 & 6 & 11.4 & 49.9 \\
QPSK 3/4 & 9 & 8.1 & 21.9 \\
16QAM 1/2 & 11.5 & 6.1 & 15.6 \\
16QAM 3/4 & 15 & 4.1 & 4.7 \\
64QAM 2/3 & 17 & 3.2 & 2.9 \\
64QAM 2/3 & 19 & 2.6 & 1.8 \\
64QAM 3/4 & 21 & 2 & 3.2 \\
\hline \hline
\end{tabular}

\section{B. Relay Station Positioning}

The initial step of this work was to obtain some indication of the maximum throughput that can be achieved where the position of the RS has a significant impact. In order to do this the topology was composed of one MR-BS, one RS, and one SS which were assumed collinear. The distance between the MR-BS and the SS was assumed fixed while the RS position was varied. The SS was put at the outside of the inner edge of the MCS zone in which throughput increase is possible (see Table 2). This specific location was chosen as it ensures the highest signal strength possible on the RS-SS link and thus maximise the achievable throughput increase. Then, the RS position was varied for different positions of the SS in order to investigate the variation in terms of throughput performance in this simple topology (Figure 4). No spatial reuse is considered and the same channel was used for MR-BS/RS communications and RS/SS communications. It is worth noting that the results are assumed symmetric with respect to the RS located at equidistance of the MR-BS and the SS.

It can be seen in Figure 5 that the maximum throughput increase possible is of $31.3 \%, 12.3 \%$ and $4.8 \%$ for an SS respectively located in the QPSK1/2, QPSK3/4 and 16QAM1/2 zone. In each case this peak is reached when the RS is using 64QAM2/3 to communicate with either the MR-BS or SS. In other words, this corresponds to the RS position where the best achievable MR-BS/RS - RS/SS links MCS pair is reached. However, this achievable improvement in the three most robust MCS region is not valid for all the possible location of the SS within each of these regions as illustrated in Figure 6.
Figure 6 depicts the different achievable throughput enhancement within the MR-BS cell by varying the SS position for different RS positions. As the SS moves from the closest point of the MR-BS to the furthest point of the MR-BS within a same MCS zone and a specific RS position the throughput gain progressively drops to eventually reach zero. This is due to the degradation of the RS/SS link quality irrespectively to the RS position within the MR-BS cell coverage. Additionally, it can be seen that when the RS is located in a MCS zone leading to a potential throughput increase in a portion of the MR-BS cell this area is maximised when the RS is at the inside of the outer edge of this MCS zone.

Figure 7 gives a general view of the results from Figure 6 . It depicts the different annuluses (i.e., zones) in which throughput increase is possible relative to the RS position. With the assumption that the MR-BS and RS use the same transmission power it can be seen in Figure 7 that no throughput enhancement is possible in a substantial portion of MR-BS cell coverage area. In these areas, there is no pair of available MCS for the MR-BS/RS link and RS/SS link which can result in a throughput improvement compared to the direct MR-BS/SS link. More specifically, it can be seen in Figure 7 that no throughput increase is possible in the first four least robust modulation scheme regions; this is in contrast to the most robust region (QPSK1/2) where up to $78.8 \%$ of this area can benefit from a throughput increase by incorporating a relay. Finally, the deployment of a relay in the network can provide throughput enhancement in a maximum of $48.7 \%$ of the total surface covered by the MR-BS with RS using 64QAM2/3 to communicate with the MR-BS.

The performance of the system is tightly related to the antenna system used. In order to provide higher throughput enhancement in a broader part of the MR-BS cell coverage an approach based on directional antenna can be considered. Each deployed RSs can be comprised of two directional antennas: a narrow beam directional antenna towards the MR-BS and a directional antenna with a larger aperture oriented towards the SSs. With such system design, significant gain can be achieved compared to an omnidirectional system. At equal transmission power higher signal quality between the MR-BS/RSs and the $\mathrm{RSs} / \mathrm{SSs}$ can be achieved with less interference and better spatial reuse.

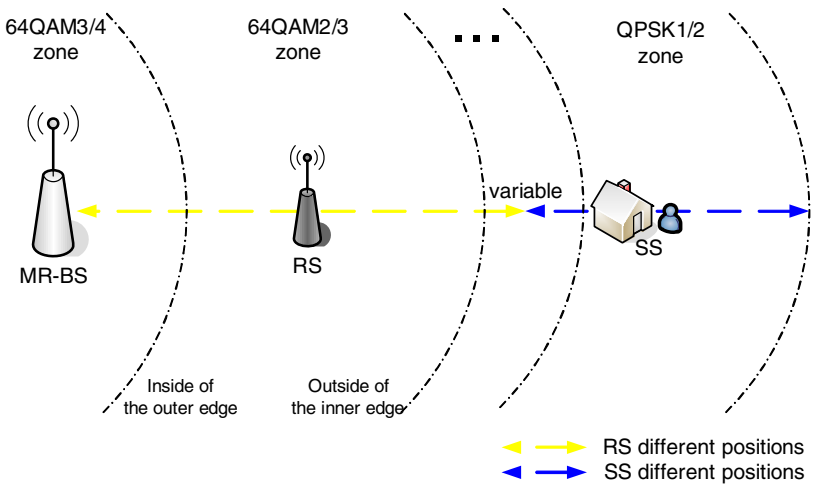

Figure 4: RS positioning - Example of the Topology 

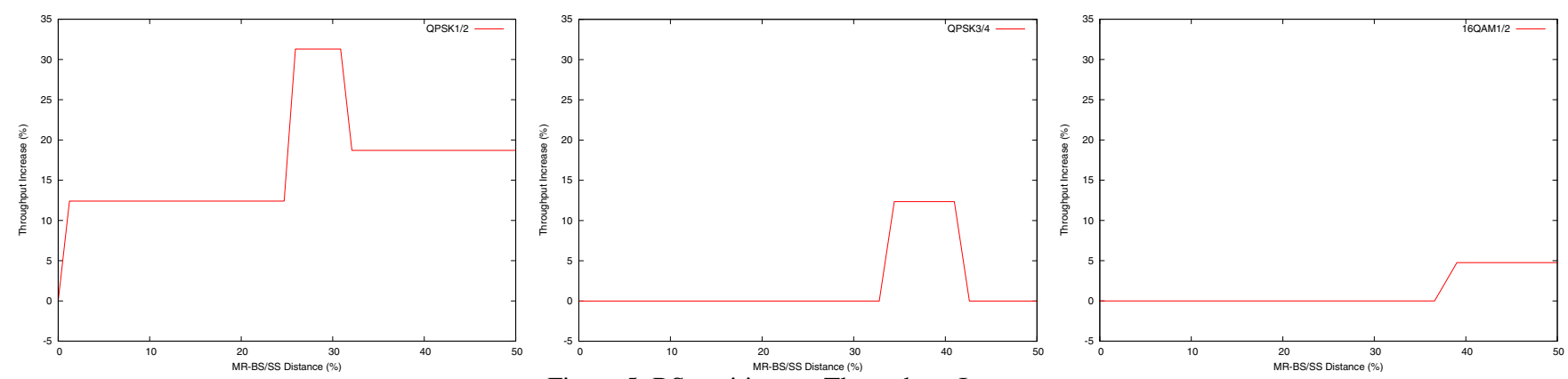

Figure 5: RS position vs. Throughput Increase

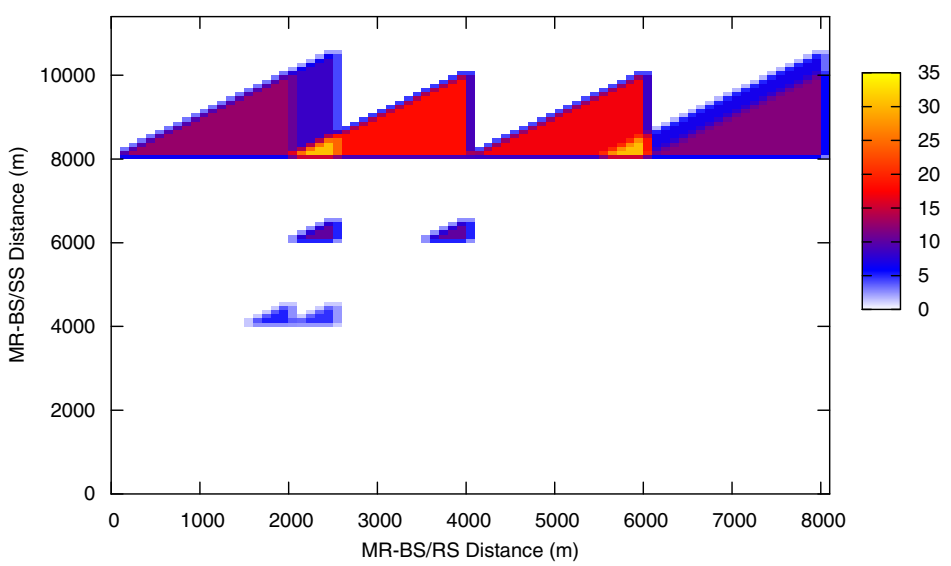

Figure 6: RS/SS position vs. Throughput Increase

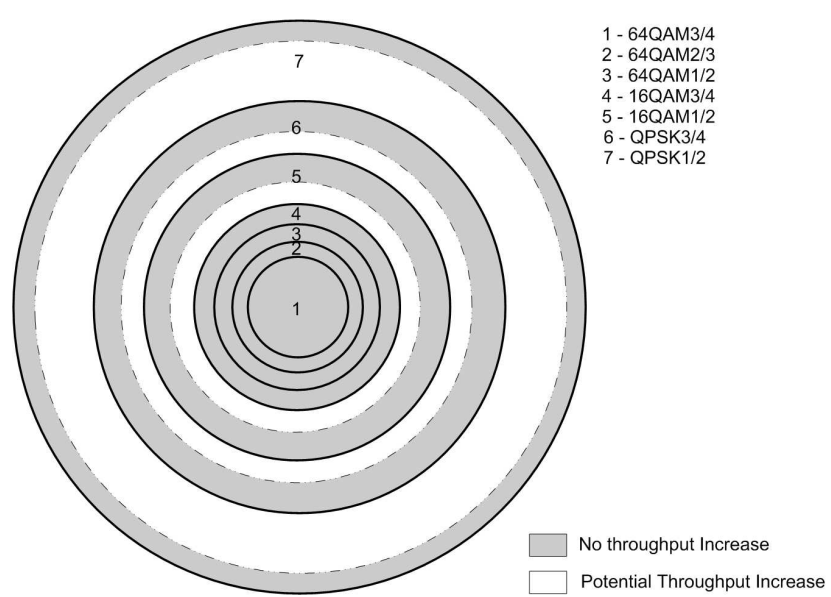

Figure 7: Potential Throughput Increase Areas in the MR-BS cell coverage Area

\section{Impact of signalling on the system throughputt}

As defined in [2], the resource allocation in 802.16 PMP system is performed each frame in a per connection basis where for each allocation a signalling message is sent by the BS to specify how to access or send the data on the medium. In [12], authors show the inefficiency of such approach in a PMP system with a degradation of the MAC efficiency of approximately $50 \%$ as the number of connections increases. Hence, there are some incentives to study the impact of the signalling relative to the resource allocation in 802.16 MMR system.

In order to analyse the impact of the signalling on the throughput of $802.16 \mathrm{j}$ system the simulations were carried out in a $10 \mathrm{~ms}$ and $20 \mathrm{~ms}$ frame sizes. The topology was composed 
of one MR-BS, one RS, and from 1 to 45 SSs located at the QPSK1/2 zone. The RS and SSs were positioned based on the results obtained above to get the maximum throughput increase. All SSs were scheduled at least once in each frame, and no data were sent in the uplink.

Figure 8 depicts the percentage of PS used for signalling in each frame. In a system using $10 \mathrm{~ms}$ frame size with the relay managing traffic for 45 connections, $7 \%$ of the system resources are used for signalling purposes where $14 \%$ in the downlink as depicts in Figure 8. This increase is due to the allocation of resource in the access zone and in the relay zone with the associated signalling messages in order to provide the necessary information on the path to access the data. It can be seen that $802.16 \mathrm{j}$ has significantly greater signalling overhead up to $90 \%$ of extra resources used compared to 802.16e in our study case. Further, as shown in Figure 9 in each case the efficiency of system in terms of throughput drops as the signalling size increase. These results highlight the inefficiency of using signalling on a per connection basis in a MMR topology. This leads in a 802.16 system to the use of a significant amount of the resources for signalling messages.

Traffic aggregation is possible on the relay link and can be easily done to save some bandwidth by reducing the signalling overhead. However, on the access link such bandwidth saving is more complex. The deployment of relay allows the use of spatial reuse which appears to be necessary to achieve significant throughput enhancement. Thus, care must be taken when designing these systems to ensure that the signalling functions only consume a limited amount of the system resources.

\section{Impact of number of RSs on overall system throughput}

This section presents the potential throughput increase depending on the number of RSs deployed in a network containing 45 SSs. It was assumed that the nodes were uniformly distributed in the area covered by the MR-BS. Hence, it was possible to determine the number of nodes that fell into the annulus corresponding to each modulation and coding scheme using the methodology described in [11] (see Table 4). A $20 \mathrm{~ms}$ frame size was assumed since the impact of the signalling on the system capacity is smaller. All the RSs were deployed uniformly at the inside of the outer edge of the 64QAM2/3 zone which represents the region in which throughput enhancement is possible for a maximum numbers of SSs in the first three most robust MCS zones.

It can be seen in Figure 10 that the system throughput increase stabilise when 4 relays are deployed. Thus, the MRBS cell is properly covered by this amount of relays and additional relays does not have a significant impact on the system capacity. However, the system throughput is only increased on the downlink by approximately $5 \%$ without any extra signalling for data traffic on the uplink. Thus, the performance gain from deploying relays under our assumptions is relatively low without any spatial reuse and with users uniformly distributed within the cell coverage.

Finally, a more appropriate system design incorporating directional antenna is likely to deliver much improved performance, but the system studied here is found severely wanting and that a cost benefit analysis of this relay solution would seem probably resulting in the relay solution being rejected in favour of a BS only solution.

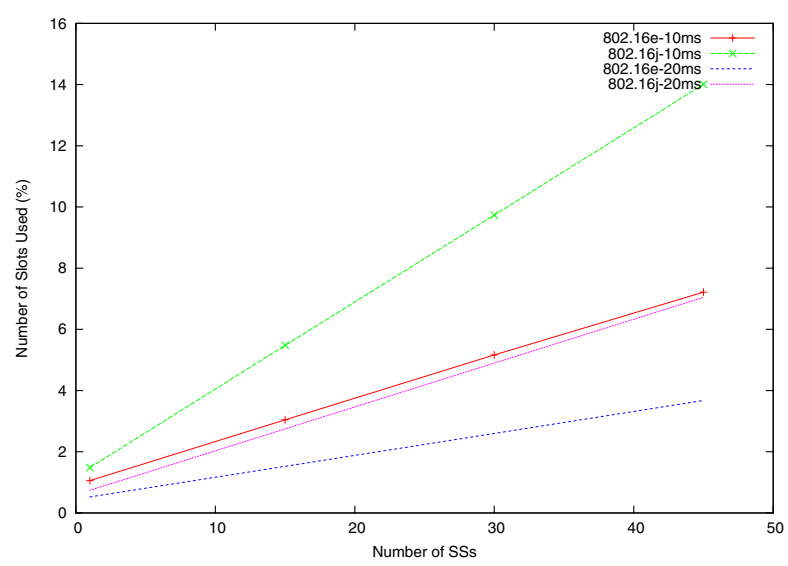

Figure 8: Signalling Size vs. Number of Users

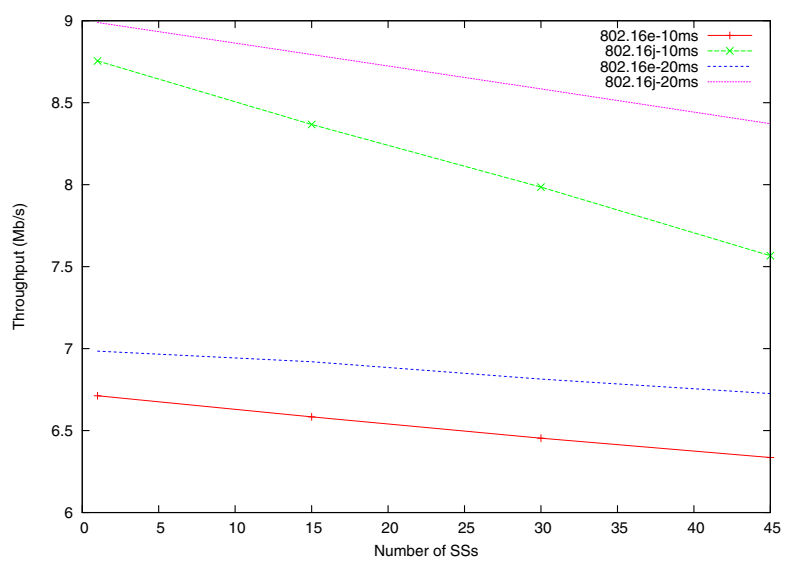

Figure 9: Throughput vs. Number of Users

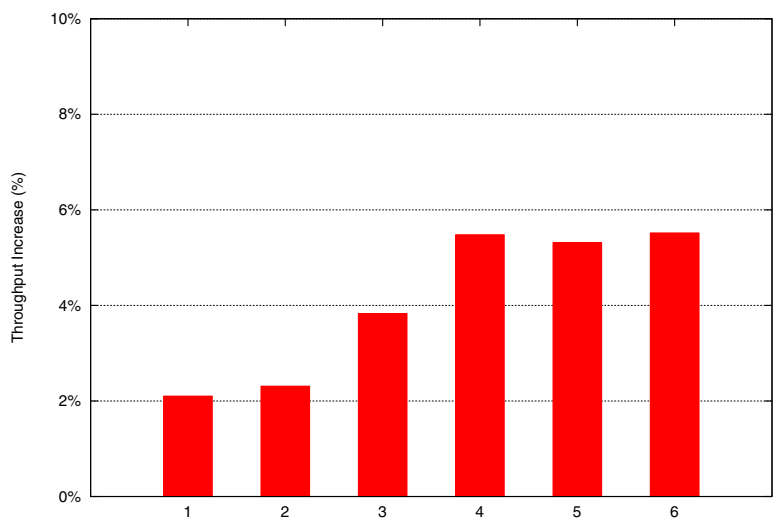

Figure 10: Throughput increase relative to the number of RSs

\section{CONCLUSION}

In this paper the system throughput enhancement that can be provided by the deployment of relays in a WiMAX network is investigated. The paper is based on the latest specifications 
of the future IEEE 802.16j MMR standard and more especially on the transparent mode for throughput enhancement purposes.

The results show first that only approximately half of the region covered by the base station can obtain increase throughput via use of RSs in an omnidirectional antenna system. Then, the paper highlights that $802.16 \mathrm{j}$ based systems introduce further signalling overhead, with almost twice that of 802.16e in the cases studied. Finally, the maximum throughput enhancement that can be expected with no spatial reuse is approximately $5 \%$ in a topology with users uniformly distributed within the cell. This work demonstrates the necessity to find ways to improve the 802.16j MMR network performance such as investigation on directional antenna and spatial reuse.

Future work will include designing a scheduling algorithm maximising spatial reuse, adding directional antenna support and a more detailed signalling messages model into the $802.16 \mathrm{j}$ simulation model.

\section{ACKNOWLEDGMENT}

V. Genc gratefully acknowledges the support of IBM and the Irish Research Council for Science, Engineering and Technology.

S. Murphy gratefully acknowledges the support of the Enterprise Ireland Commercialisation Fund.

\section{REFERENCES}

[1] IEEE, IEEE Std 802.16-2004, "IEEE Standard for Local and Metropolitan Area Networks, Part 16: Air Interface for Fixed Broadband Wireless Access Systems", October 2004
[2] IEEE, IEEE Std 802.16e-2005, “IEEE Standard for Local and metropolitan area networks Part 16: Air Interface for Fixed and Mobile Broadband Wireless Access Systems. Amendment for Physical and Medium Access Control Layers for Combined Fixed and Mobile Operation in Licensed Bands", February 2006.

[3] IEEE 802.16j MMR Work group, http://www.ieee802.org/16/relay/

[4] IEEE, IEEE Baseline document v4 for draft standard, "Part 16: Air Interface for Fixed and Mobile Broadband Wireless Access Systems, Multihop Relay Specification”, May 2007.

[5] M. Nohara, J. Puthenkulam, M. Hart, M. Asa, J. Cho, IK. Fu and al, IEEE 802 Tutorial: 802.16 Mobile Multihop Relay, March 2006. Available at http://www.ieee802.org/16/sg/mmr/index.html.

[6] L. Erwu, W. Dongyao, L. Jimin, S. Gang, J. Shan, "Performance Evaluation of Bandwidth Allocation in 802.16j Mobile Multi-hop Relay Networks", VTC2007-Spring, Dublin.

[7] Zhifeng Tao, Koon Hoo Teo, Jinyun Zhang, "Aggregation and Concatenation in IEEE 802.16j Mobile Multihop Relay (MMR) Networks", Mobile WiMAX Symposium, 2007.

[8] Shiang-Jiun Lin, Wern Ho Sheen, I-Kang Fu, Chia-chi Huang, "Resource scheduling with directional antennas for multi-hop relay networks in Manhattan-like environment", Mobile WiMAX Symposium, 2007.

[9] B. Lin, P. Ho, L. Xie, X. Shen, "Optimal Relay Station Placement in IEEE 802.16j Networks”, IWCMC, August 2007.

[10] QualNet simulator, http://www.scalable-networks.com/

[11] C. Hoymann, "Analysis and Performance Evaluation of the OFDMbased Metropolitan Area Network IEEE 802.16," Comp. Net., vol. 49, no. 3, Oct. 2005, pp. 341-63.

[12] A. E. Xhafa, S. Kangude, and X. Lu, "MAC performance of IEEE 802.16e," in Proceedings of IEEE 62nd Vehicular Technology Conference, September 2005 